\title{
Could we now convince Einstein?
}

\author{
LUIGI ACCARDI ${ }^{1}$
}

\author{
Centro Vito Volterra, Università of the Studies of Rome " Tor Vergatà ' \\ WEB-page: http://volterra.mat.uniroma2.it, \\ Quantum Theory: reconsideration of foundations-3 \\ Vaxjo, June 6-11 (2005)
}

\begin{abstract}
The present conference takes place in the same year that celebrates the centenary of Albert Einstein. Hence it is a good occasion to reflect on those problems which have been at the core of Einstein's intellectual activity. Undoubtedly the foundation of quantum mechanics (QM) is one of these problems. It is known that Einstein was never convinced by the interpretation of quantum mechanics accepted, in his times and still now, by the majority of physicists. The fact that he was sharing this skepticism with people like Schrödinger and, most of all, the fact that no convincing answer, to the doubts of these people, had emerged in a more than half a century old debate, helped in keeping alive the attention of a growing number of people on this problem.

The crucial issue is that the standard interpretation of QM has some physical implications which are experimentally verifiable and which, for several years, have been thought to be incompatible with relativity theory (the so-called "quantum nonlocality"). On the other hand alternative, more intuitive, interpretations (such as the ensemble interpretation) seemed to be ruled out from very well confirmed experimental data.

The way out from this impasse has required a deep analysis of the connections between mathematics and physics as well as the emergence of new ideas both in mathematics (non-Kolmogorovian probabilities) and in physics (the theory of adaptive systems).

The Einstein centenary is a good occasion for a short survey of these developments with the goal of answering the intriguing question posed in the title of the present paper.
\end{abstract}

\section{Introduction}

The debate on the foundations of quantum theory is at the center of the reflections on the philosophy of science since more than three quarters of century.

In these years practically all the greatest physicists and mathematicians, and the most important philosophers of science have given life to a debate that has few equals in the history of the human thought for depth, width and subtlety of the arguments involved.

Recently it has been claimed that the fall-out of this debate can also influence technology, or even industry, through catch words such as quantum cryptography, quantum computer, teleportation, etc. ....

This may well be true. However I feel that the reason why the most qualified minds in philosophy, physics, mathematics are dedicating their thoughts, since almost one century, to the interpretative problems of quantum mechanics is to be looked for not so much in these virtual applications to technology and industry, as in the strong fascination arising from the purely intellectual and abstract challenge concerning the problem of knowledge and, in particular, the limits to the human attempts of building a coherent picture of the physical world.

The present knowledge of the physical world is based on two fundamental theories, relativity and quantum mechanics, whose synthesis is still far from the horizons of contemporary science.

These two theories are quite different in their structure: relativity is exact, deterministic and based on a very intuitive, mainly geometrical, mathematical formalism. Probability may enter in it only through incomplete knowledge. On the contrary quantum mechanics is intrinsically probabilistic and based on an abstract, functional analytic, formalism which, as recognized by Heisenberg himself in the early years of its development, preceeded its physical interpretation and is "far from the intuition" (this is a very peculiar feature of the historical development of quantum theory with respect to all other physical disciplines).

But our way of thinking of the physical phenomena is based on intuition, therefore something "far from the intuition" is also beyond (para') the common opinion (doxa). In this sense the Greek roots of the words fully justify the term "paradox".

\footnotetext{
19accardi@volterra.mat.uniroma2.it
} 


\subsection{The two roots of the quantum paradoxes}

The notorious "paradoxes of quantum mechanics" emerged during the long and not always straight path which, from the attempts to explain the experimental data coming from the atomic and sub-atomic world, led to the present interpretation of the theory.

These paradoxes seemed to indicate the existence of unavoidable contradictions between:

(i) a realistic vision of the world, in which the objects have objective and pre-determined properties.

(ii) the experimental results of quantum theory

(iii) the theory of relativity and in particular the principle of locality.

The debate on the interpretation of quantum theory rotated since the beginning around these three basic conceptual poles but, in different times, the main emphasis shifted from some of these pole to others.

In the approximatively 80 years passed from the establishment of the new quantum theory to nowadays, for about 40 years, say from 1925 to 1964, the paradigmatic example to illustrate the quantum paradoxes was the two-slit experiment; in the remaining 40, say from 1964 to now, attention shifted towards the EPR correlations.

In the first phase of the debate, relativity and the validity of the experimental results of quantum theory were given for granted and emphasis was on realism: according to the orthodox interpretation, given these premises, a non observed particle cannot have a definite position.

In the second phase of the debate, relativity, the validity of quantum theory and realism were put on the same ground and emphasis was on locality: if you accept realism and the experimental results of quantum theory, then you must abandon locality, hence relativity, and accept the "spooky action at distance".

The dramaticity of the situation was sharpened from the fact that it seemed there were no ways out from this alternative: from one side the logical reasoning, leading to the paradoxes, seemed to the majority of physicists unattackable: simple mathematical arguments, people argued, show that every realistic model of the world that respects the principle of locality must satisfy some inequalities.

On the other hand, the most accurate experiments, that in these years continue to be repeated and to receive confirmations, demonstrate that such inequalities are violated in nature.

There are two main classes of such experiments: those of "interference-type" (double slit, SQUID, macromolecules, ...) and those of "correlation-type" (spin, polarization, ...). The former refers to the two-slit experiment, the latter to the EPR-type experiments.

The opinion of the majority of physicists, who have published papers on such contradictions is that the only way out is to throw in the sponge and to recognize the incompatibility of quantum theory with "local realism": no classical system can, with local and independent choices, reproduce the EPR (Einstein, Podolsky, Rosen) correlations or the two-slit probabilities.

The situation begun to change towards the end of the 1970's, when it was discovered that the deduction of both the above mentioned inequalities relied on a common hidden hypothesis, of purely mathematical nature.

\subsection{The starting point of quantum probability}

There is a huge literature discussing the meaning and the implications of both Bell's and the 2-slit inequalities.

It is therefore somewhat surprising the fact that the common mathematical root of these inequalities remained unnoticed until 1981.

The discovery, in [Ac81a], [AcFe81] that both the 2-slit inequality and the Bell inequality are two different aspects of the same mathematical phenomenon was the starting point of quantum probability.

More precisely: both the 2-slit inequality and Bell's inequality are necessary conditions for the existence of a Kolmogorovian model for a set of statistical data coming from different and mutually incompatible experiments: conditional probabilities in the 2-slit case; correlations in the Bell case.

The above mentioned remark was the root of the basic intuitions of quantum probability, namely:

(i) There exist many inequivalent models of laws of chance (probability) just as there exist many inequivalent models of laws of space (geometry)

(ii) It is possible to distinguish, by means of experiments, which models can describe a preassigned set of statistical data

(iii) it is necessary to study systematically, both in their mathematical aspects and in their empirical origins (i.e. not only physical, but also biological, medical, sociological, economical, ...) these new non Kolmogorovian models.

The above statements, especially (i) and (ii), are very strong. Can one prove them in the sense of deducing their necessity from the combination of mathematical theorems and experimental data?

The answer is yes and the basic tool for this proof is given by the statistical invariants. In the following we will illustrate this notion through a few basic examples. 


\subsection{The statistical invariant of the two slit experiment: a quantum proba- bilistic analysis}

We follow Feynman ([Feyn51] or [FeLeSa66]) in the exposition of this experiment and [Ac83a] for the calculation of the statistical invariant.

A source emits microscopic particles which can pass a first screen through two slits, labeled 1 and 2 , and are collected on a second screen. Denote $X$ an arbitrary region on this second screen.

The experimentally measurable statistical data are the probabilities:

$P(X)=$ probability of hitting the region $X$ of the screen

$P(X \mid 1)=$ probability of hitting $X$ when the slit 2 is closed

$P(X \mid 2)=$ probability of hitting $X$ when the slit 1 is closed

Definition 1 The statistical data $P(X), P(X \mid 1), P(X \mid 2)$, admit a Kolmogorov model if there exists a probability space $(\Omega, \mathcal{F}, \mu)$ and events, still denoted $X, 1$ and 2 and identified to subsets of $\Omega$, such that:

$$
\begin{gathered}
P(X)=\mu(X) \\
P(X \mid 1)=\frac{\mu(X \cap 1)}{\mu(1)} \\
P(X \mid 2)=\frac{\mu(X \cap 2)}{\mu(2)}
\end{gathered}
$$

Remark The above definition means simply that in the classical, Kolmogorovian model the conditional probabilities $P(X \mid 1)$ and $P(X \mid 2)$ must be given by the usual Bayes' formula.

The crucial fact to be noted here is that the statistical data $P(X), P(X \mid 1), P(X \mid 2)$, have a well defined experimental meaning independently of any mathematical model (just as the angles in Gauss' triangle). Therefore the existence of a Kolmogorov model for them cannot be postulated, but it must be proved. Exactly as one cannot postulate the existence of an Euclidean triangle determined by three empirically measured angles, but one must use Gauss' criterium to check if such a triangle exists or not.

Proposition 1 The statistical data $P(X), P(X \mid 1), P(X \mid 2)$ admit a Kolmogorov model if and only if the given probabilities satisfy the constraints

$$
0<\frac{P(X)-P(X \mid 2)}{P(X \mid 1)-P(X \mid 2)}<1
$$

Proof. One solves the system (1), (2), (3), in the unknowns $\mu(X \cap 1), \mu(1), \mu(X \cap 2), \mu(2)$, and one imposes the condition that the solutions are in $(0,1)$.

Remark If one changes the statistical data in (4), i.e. the probabilities, so that the constraint (4) is still fulfilled, then the property of admitting a Kolmogorovian model will remain unaltered.

In this sense we say that the relation (4) is a statistical invariant for the set of statistical data $P(X), P(X \mid 1)$, $P(X \mid 2)$ with respect to the Kolmogorovian model.

We can see that, if the empirical data $P(X), P(X \mid 1), P(X \mid 2)$ have been obtained in three different experiments on three different samples, then condition (4) is by no means a tautology on the relative frequencies: its validity is an experimental fact. If it holds, then a Kolmogorov model exists and the identity

$$
P(X)=\mu(1) P(X \mid 1)+\mu(2) P(X \mid 2)
$$

can be satisfied with an appropriate choice of the probability $(\mu(1), \mu(2))$.

The experiment discussed by Feynman (and before him by Bohr, Einstein, Heisenberg, ...) shows that in some cases (5) is not satisfied so that a Kolmogorov model for these data cannot exist.

Summing up: the 2-slit experiment, whose conceptual implications were first discussed in the 5-th Solvay conference in 1927, provides the first example of a simple set of statistical data, coming from different but related experiments, which do not admit a Kolmogorovian model.

\subsection{The statistical invariant of the EPR type experiments: a quantum probabilistic analysis}

According to the majority interpretation of quantum mechanics some properties of physical objects have only a virtual existence which becomes actual with the act of measurement. Moreover this transition virtual-actual is a physical phenomenon in the sense that the measurement does not unveil to our knowledge a pre-existing physical reality, but "creates" such a reality with probabilities determined by the wave function. 
In 1935 Einstein, Podolsky and Rosen (EPR), developing an earlier idea of Popper, published a paper [EPR35] which pointed out some difficulties in this interpretation.

More precisely, by a clever use of some conservation laws, they proved that if one insists in the claim that the act of measurement "creates" a reality, then one could create such a reality instantaneously at distance, thus contradicting the locality principle. This is the notorious "quantum non locality".

In 1964 Bell proved a variant of the two-slit inequality [Bell64], related to the EPR type experiments, like the original two-slit inequality also Bell's inequality can be experimentally checked.

In probabilistic language Bell's inequality can be formulated as follows:

Theorem 1 Let $(\Omega, \mathcal{F}, P)$ be a probability space and let $A, B, C$ be any three random variables defined on $\Omega$ and taking values in the interval $[-1,1]$. Then the following (Bell) inequality holds:

$$
|E(A B)-E(B C)| \leq 1-E(A C)
$$

Proof Simple exercise.

The physical implications of Bell's inequality can be formulated in the language of quantum probability as follows:

Corollary 1 There exist triples $a, b, c$ of unit vectors in $\mathbf{R}^{3}$ for which it is not possible to find six $\{-1,+1\}-$ valued random variables $S_{x}^{j}(x=a, b, c ; j=1,2)$ on the same probability space $(\Omega, \mathcal{F}, P)$ whose correlations are given by:

$$
E\left(S_{x}^{1} \cdot S_{y}^{2}\right)=-x \cdot y \quad ; \quad x, y=a, b, c
$$

(where, for $x, y \in \mathbf{R}^{3}, x \cdot y$ denotes the Euclidean scalar product.)

Proof Choose

and show that

$$
a=0 \quad ; \quad b=\frac{\pi}{4} \quad ; \quad c=\frac{\pi}{2}
$$

$$
A:=S_{a}^{1} \quad ; \quad B:=S_{b}^{2} \quad ; \quad C:=S_{c}^{1}
$$

violate Bell's inequality. The proof is concluded using the fact that (7) implies that:

$$
S_{c}^{1}=-S_{c}^{2}
$$

Summing up: it is not true that the only hypotheses of realism and locality imply Bell's inequality. On the contrary, the proof of this inequality involves an additional mathematical hypothesis, put in light for the first time by quantum probability. Namely the existence of joint probabilities for triples of spin observables.

Because of Heisenberg principle these, contrarily to what happens for the joint probability of pairs of observables, are not experimentally observable.

In particular Bell's inequality does not add anything to the conclusion of the two slit experiment since in both cases the essence of the problem not is non-locality, but non-Kolmogorovianity.

In fact in both cases the only rationally justified conclusion is that: they exist sets of statistical data, coming from mutually incompatible experiments, that cannot be described by a single Kolmogoroviano model.

Such a conclusion is definitively not amazing for a classical probabilist.

Moreover at the light of what just said it is somewhat surprising that, neglecting the fact that the 2-slit inequality preceeded Bell's of almost 40 years, some authors insist to call these type of conditions "Bell's type inequalities" rather than "2-slit type inequalities".

Starting from 1981 the quantum probability point of view, that Bell's inequalities are necessary conditions for the existence of a Kolmogorovian model for a set of correlations coming from different and mutually incompatible experiments, was debated in several papers and international conferences.

Starting from 1982 this point of view begun to be adopted by a larger and larger group of experts on the foundations of quantum mechanics (cf. for example [Fine82a], [Merm82a], [Merm82b], [Pito89], [Belt91], [BeDelNMa93], [BeCa94], [Volo00b], [HePhi01a], [HePhi01b], [FilSvoz04], ...).

Presently quantum probability $(\mathrm{QP})$ is suffering from the following schizophrenic situation:

- some say QP did not discover anything because the physicists always thought that way.

But, if this is true, then how to explain the huge literature on Bell's inequality and "quantum nonlocality"?

- some say QP is completely wrong and that the essence of the matter is non locality (cf. a recently appeared book by Zeilinger).

But, if this is true, then what is wrong with the Theorem proved in section (4.2)?

The difficulty of finding a rational answer to such questions suggests that probably they have to do with sociology rather than with science. 


\section{Bell's interpretation of the Bell inequality: non locality}

In order to emphasize the difference between the quantum probabilistic interpretation of the Bell inequality and Bell's original one, let us briefly summarize the latter.

According to Bell, the inequality (6) is necessarily implied by the locality principle according to which no physical interaction can propagate with a velocity higher than the speed of light in the vacuum.

But, according to experiments: the EPR correlations violate Bell's inequality.

Conclusion: if Bell's idea, that his inequality (6) is a necessary consequence of the locality principle, is correct, then quantum mechanics is non-local because the quantum mechanical experimental data violate the inequality (6).

But the locality principle, is one of the pillars of relativity theory.

Therefore: if Bell's idea is correct, then QM contradicts relativity.

Thus we are faced with the following dramatic question:

Is it true that the two basic theories of contemporary physics

- the theory of relativity

- quantum mechanics

are mutually contradictory?

In the past 30 years the answer to this question, accepted by the majority of physicists has been:

yes they are mutually contradictory. Moreover this can be proved by theory and confirmed by experiments.

Notice that the development of the experimental techniques has allowed to extend Bell's interpretation also to the two-slit experiment in fact one can now realize this type of experiments with a macroscopic separation between the two interference paths.

The mutual contradiction between quantum mechanics and relativity theory is a very strong statement and it is precisely this statement that explains the huge literature on both the two-slit and Bell's inequality: no physicist would have been interested in subtle technical differences between conditional and unconditional probabilities (or correlations) were it not for the nightmare of this contradiction.

\subsection{The statistical invariants of classical models}

Given the experimental data the existence of a Kolmogorov model is equivalent to the existence of a positive normalized solution of a set of linear equations.

These equations were first deduced in [AcFe81] and they were applied to construct examples showing that non Kolmogorovian transition probabilities can be obtained from the statistics of single quantum systems.

This result was later extended in the paper [Ac85] with the construction of single particle statistical correlations differing only in the sign from those of the singlet state.

This example brought further evidence against the pretension of Bell and other authors that the singlet correlations are an indication of the nonlocality of quantum theory:

in fact, since these correlations concern statistical data related to single particles and yet satisfy similar inequalities as Bell's (leading to similar conclusions, i.e. the non existence of joint probabilities), it follows that the essence of these inequalities cannot be the existence of instantaneous correlations among different particles in the same singlet pair, because in this case no pairs are involved.

\subsection{The "parallel postulate" for probability theory}

Having realized that quantum probability is a non classical model for probability theory, a natural question to be asked was: which of the classical axioms of probability are violated in quantum probability? The answer to this question was given in the paper [Ac81a] where the role of the Bayes axiom as a hidden axiom of probability theory was first pointed out.

The implicit axiom, which played for probability theory the role of the parallel postulate in geometry, is Bayes' elementary definition of conditional probability. For example this axiom is not included in Kolmogorov's axiomatization [Kolmo33], where the notion of conditional probability is introduced as a definition rather than as an independent notion, as now we understand it is.

In fact the paper [Ac85] (Section (5.)) contains a mathematical analysis of Bayes' formula, defining classical conditional probability, which shows that it is equivalent to five, mutually independent, axioms which are by far not self-evident. The same paper discusses several examples of violation of these axioms in the quantum probabilistic model. These examples are not artificially built but they correspond to experiments which have been done in the first years of quantum theory and which are now considered of a routine nature (e.g. SternGerlach type experiments). 
In the paper $[\mathrm{Ac} 81 \mathrm{~b}]$ it was pointed out that von Neumann's formula for the quantum measurement processs is equivalent to Bayes' formula plus a "Markovianity" assumption: the difference between von Neumann's formula and the usual quantum formula for superposition states is given precisely by the interference terms.

\subsection{Statistical invariants and hidden variables}

We have seen that quantum probability was born, at the end of the 1970's, with the proof of the fact that the problems risen from the two slit experiment and from Bell's inequality have the same mathematical root, i.e.: the existence of sets of statistical data, coming from mutually incompatible experiments, which cannot be described by a single Kolmogorov model [Ac81b], [Ac81a], [Ac81d].

While the common mathematical root of the two experiments, crucial for all interpretative difficulties and all the apparent paradoxes of quantum theory, had never been observed before quantum probability, the question, whether there might exist some kind of "classical model for quantum theory", was widely debated in the huge literature existing on the foundations: the whole literature on hidden variable is centered on this problem.

However it is enough to glance, even superficially, at this literature, to become aware of the fact that, under the common designation "classical model for quantum theory", one could find a multiplicity of totally different models (cf. e.g. [Jamm74] or [Ac86]) whose only common feature is the fact that each of them introduces several assumptions whose physical meaning and justification are rarely discussed.

All the no-go theorems on the existence or non existence of hidden variables followed the pattern of the first of these theorem, due to von Neumann [vNeum32], and so their conclusions are restricted to the existence or non existence of an "isomorphism" between the quantum model and some classical one.

Since there is no canonical notion of "isomorphism" between different models, these conclusions were very unstable with respect to their definition of this notion of "isomorphism": it became clear with the proliferation of these theorems and of critiques to them that a small change in the assumptions might lead to completely opposite conclusions.

The contribution of quantum probability to this problem has been to shift the attention from the idea of "isomorphism between models" to the notion of "constraints among experimental data and the possibility of a given classical model": the Kolmogorov representability (or not), of a set of statistical data, can be decided only on the basis of experimental data, without any additional mathematical assumption.

The statistical invariants are the mathematical tools that allows to carry out this decision. The term "statistical invariant" was coined in analogy with the term "geometrical invariant". In fact the path which lead to the new probabilities presents many analogies with he path which lead to the new geometries. In the following section we briefly discuss these analogies.

\section{Analogies between the development of quantum probability and of non Euclidean geometry}

The term "quantum probability" although used since the early days of quantum theory, acquired a precise mathematical and physical meaning in the late 1970's, with the discovery of the "statistical invariants".

These play, for probability, the same role that the geometrical invariants play for geometry and physics, i.e. they are experimentally measurable quantities which allow to distinguish among different mathematical models.

There are other remarkable analogies between the historical development of geometry and that of probability.

The development of both disciplines has been strongly influenced by the parallel development of the natural sciences (in first place physics) and of technology.

In geometry it took several thousands years to realize the existence of a multiplicity of mathematical models of space.

In probability it took only a few hundred years to realize the existence of a multiplicity of mathematical models of the laws of chance, but in a period in which the rate of scientific development had undergone a drastic acceleration.

In geometry non Euclidean models had been in use, for practical applications (spherical geometry) or for theoretical purposes (projective geometry), for a long time before it was realized by Gauss that the differences among these models might be intrinsic and physically measurable.

With the hindsight coming from this discovery, several results, which were originally proved within the Euclidean model, could be re-interpreted as criteria, distinguishing one model from another. Typical examples are Euclid's theorem on the sum of the inner angles of a (Euclidean) triangle, Desargues or Pascal's theorems in projective geometry, .... 
Almost the same happened with probability: physicists used a non-Kolmogorov probabilistic model, i.e. the quantum probabilistic model, several decades before it was recognized that some of the basic elementary probabilistic laws might be model dependent statements.

Once this was recognized, in the late 1970's, several theorems of classical probability, like the Kolmogorov compatibility conditions for joint probabilities, or some of their several ancestors due to different mathematicians, from Laplace, to Boole, or some of their corollaries like Vorobiev's criteria, could be interpreted as criteria to distinguish among different probabilistic models.

After the non-Euclidean revolution geometry ceased to be the study of the Euclidean model to become the study of possible models of the laws of space and astrophysics became a kind of experimental geometry.

Similarly, after the change of perspective due to quantum probability, probability is no longer the study of the single Kolmogorov model, but the study of the possible models for the laws of chance. Statistics, on the other hand, is becoming the ideal experimental ground to check for the existence of non Kolmogorovian models outside the quantum domain, for example medicine, economics, sociology, politics, ... .

Both in probability and in geometry this development was accompanied by a strong trend towards algebraization from one side and by a stronger relation with experimental data from the other.

In both cases the overcoming an historical prejudice opened fascinating perspectives to theoretical and experimental investigations. Geometry has already gone very far in this direction. Probability is just beginning. In the following we will discuss some of these developments which are particularly relevant to the problem of the foundations of QM.

For lack of space we will not discuss:

- advanced mathematical aspects of quantum probability: connections with classical probability, functional analysis, theory of operators, algebra, geometry, ... (for these topics we refer to the [QP - PQ] series in the bibliography below, which includes now more than 20- volumes)

- concrete physical applications such as quantum statistical mechanics of equilibrium and non equilibrium phenomena, solid state physics, quantum field theory, ... (for these topics we refer to the recent monographs [AcLuVo02], [AcKo00b]).

\subsection{The statistical invariants of the quantum model}

Having introduced the notion of statistical invariants for the classical model, it was natural to conjecture that such invariants should exist also for the quantum probabilistic model.

The paper [AcFe81] contains the first explicit computation of examples of such invariants (concerning triples of two-valued observables).

As an application of this, it was shown that, in the quantum case, the statistical invariants are fine enough to distinguish between real and complex Hilbert spaces uniquely in terms of the (experimentally observable) conditional probabilities. This result solved a long standing open problem in the foundations of quantum theory, namely to give a physical justification of the emergence of complex rather than real Hilbert space in the standard quantum mechanical model.

The proof of this result was simplified by Gudder and Zanghi [GuZa84] and further improved in [Ac86], where the result was extended from three to an arbitrary number of two-valued observables (in this extension a new invariant, of cohomological nature, arises).

The technique of the statstical invariants also allows to solve the converse of the problem that arises in connection with the 2-slit-type inequalities (including Bell's): we have seen that these inequalities allow to prove the existence of simple experimentally measurable statistical data which admit a quantum probabilistic but not a classical probabilistic model.

Are there examples of statistical data which admit a classical but not a quantum probabilistic model?

The first such example was built in [AcFe81], but it was purely computational and did not give much insight. A more interesting example was given in [Ac82c] (cf. Problem (I.a)) where it is shown that the (bi-stochastic) transition matrix $P=\left(p_{i j}\right)$ of any (even non homogeneous) random walk does not admit a (pure) quantum model, i.e. there exist no unitary matrix $U=\left(u_{i j}\right)$ satisfying

$$
p_{i j}=\left|u_{i j}\right|^{2} \quad ; \quad \forall i, j
$$

Recently, this result has been extended by Meyer [Mey96], who proved that a non symmetric random walk is unitarily implementable if and only if it is a one-side shift (i.e. shift along a fixed direction), namely, it is a totally deterministic process.

In this connection it is interesting to observe that, if one weakens the requirement from "unitarily implementable" to "isometrically implementable", then every Markov chain becomes implementable in this sense: 
this fact has been recently exploited in [AcFi03] for the construction of "entangled random walks" (cf. the recent note [Miyad05] for a justification of this name).

A further natural problem in this connection is the construction of probabilistic models which are inequivalent both to the classical and to the usual quantum probabilistic model. Such examples are constructed, in section (5.) of $[\mathrm{Ac} 82 \mathrm{c}]$, with techniques of differential geometry and are related to the gauge theories: the statistical invariant in such models turn out to be related to the geometrical invariants, i.e. to the curvature of the connection.

\subsection{The deduction of the quantum model from physical requirements}

The theory of statistical invariants allows to discriminate between the classical probabilistic model and others (e.g. the quantum one), but it does not throw light on the problem of deducing these models from physically meaningful axioms, in particular it does not allow to answer the following questions:

(i) which new physical requirements imply the introduction of non classical models of probability?

(ii) can one deduce the quantum formalism from physically meaningful axioms?

Only after that such a deduction has been achieved one can claim to have achieved a deeper understanding of the origins of the differences among the various probabilistic models.

On the second question there exists an immense literature in which one can distinguish several different lines of research, among which the largest one, in terms of number of papers produced, is the "quantum logics". However, from the point of view of the conceptual clarification of the problem, the results obtained from this abundant literature are somewhat disappointing.

The first, and presently the only deduction of the quantum formalism from physically meaningful axioms was obtained in the papers [Ac82a], [Ac82c] and subsequently elaborated in the papers [Ac95], in terms of a new axiomatization of probability which adopts a point of view quite different from those of Kolmogorov, De Finetti, ... .

This new axiomatization of the theory of probability is not based, as the former ones, on the notion of event (independent from every interaction) but on that of measurement (that instead depends on an interaction). The notion of event is then obtained as a particular case.

The axioms lead to an algebraic structure (the Schwinger algebras) and the classification of the probabilistic models is achieved by solving the equations for the structure constants of these algebras.

The paper deals with the finite dimensional case (observables with only a finite number of values) but the infinite dimensional extension can be achieved with the same techniques.

An explicit list of the axioms together with the extension of this result to observables with countably many values was described in [Ac95a].

Those who love the a posteriori reconstructions could conclude that the passage from the new axiomatization of probability to the "urns-chameleon" metaphor is immediate.

In effects the color of one little ball in a urn is a typical example of event (independent from the interaction), while the color of chameleon on the leaf or the log is a typical example of response to one measure (dependent on the interaction).

Although correct from a logical point of view, this conclusion does not reflect the real historical course of the events. In fact the passage from the new axiomatization of probability theory to the statistics of responses required more than 11 years.

\subsection{Complementary pairs}

The paper [Ac82c] also contains an attempt, the first one to my knowledge, of giving a mathematical formulation of Bohr's distinction between the notion of incompatibility and complementarity for pairs of observables.

The intuitive idea underlying this formulation was that, according to the basic philosophy of quantum probability, this difference should be encoded into measurable statistical properties, i.e. into the transition probabilities.

Since the transition probabilities between two compatible (maximal) observables $A, B$ are deterministic (i.e. can take values only 0 or 1 ) while for general incompatible observables they can take arbitrary values, the complementary pairs should be characterized by the property that a maximal quantum information on one of the two, i.e. the precise knowledge of one eigenvalue, should correspond to zero information on the other. In terms of transition probabilities this means that, if $\varphi_{j}$ are the eigenvectors of the observable $A$ and $\psi_{k}$ the eigenvectors of the observable $B$, then the knowledge of $\varphi_{j}$ should induce the uniform distribution on the eigenvalues of $B$, equivalently, if the observables are non degenerate and $n$-valued:

$$
\left|\left\langle\varphi_{j}, \psi_{k}\right\rangle\right|^{2}=\frac{1}{n} \quad ; \quad \forall j, k
$$


The extension to observables with continuous spectrum led to the nice formula, proved in the same paper:

$$
\operatorname{Tr}\left(E_{I}^{Q} E_{J}^{P}\right)=2 \pi|I| \cdot|J|
$$

where $E_{I}^{Q}$ (resp. $E_{J}^{P}$ ) are the spectral projections of the position (resp. momentum) operator and $I, J$ are arbitrary intervals in $\mathbb{R}$ and $|I|,|J|$ their Lebesgue measures.

A natural equivalence relation among complementary pairs was then introduced and a direct (but not very inspiring) calculation shows that inequivalent pairs exist even in finite dimensions. The problem of classifying such pairs up to equivalence was then stated in the paper as one of the interesting open problems in quantum probability (cf. formula .3 in Problem (I.f) of [Ac82c]).

This problem was later studied by several authors: Kraus [Kra87] produced examples of inequivalent complementary pairs in the finite dimensional case; Maassen, who attended the 1982 Mondragone conference where this formula was first presented, together with Uffink gave a nice characterization of complementary pairs, in finite dimensions, in terms of entropic uncertainty relations [MaasUff88]; the trace formula above, in the continuous case, was further investigated by Lahti [Lahti85] and Lahti and Ylinen [LaY187]; however the real breakthrough in our understanding of the origin of inequivalent complementary pairs came from Cassinelli and Varadarajan [CasVar02] who constructed the first example of such pairs in the continuous case exploiting the existence of two inequivalent notions of Fourier transform on the real line.

The interest on complementary pairs was recently revived in literature on quantum information, where they have been re-baptized "mutually unbiased pairs", and active research on this topic is now being developed in this direction (cf. [Bengt03], [Bengt04]).

\section{Implications for the locality problem}

In parallel with the matematical developments (calculation of statistical invariants for several types of models, construction of the new axiomatization of probability, ...) the implications of these developments for the problem of quantum paradoxes were developed. These were discussed among other places, in the conference "The waveparticle dualism" organized in 1982 in Perugia by S. Diner, G. Lochak and F. Selleri, whose proceedings appeared two years after [Ac84]. These implications can be synthesized in the review article [Ac86], which compares several approaches to the problem of the so-called quantum paradoxes and provides a sufficiently clear picture of the quantum probability approach at the beginning of the 80's.

But the technical developments were not sufficient: it was necessary a new idea that could explain in intuitive and simple way the physical reasons of the emergency of non Kolmogorovian probabilistic models. The problem, that remained opened in the 80 's, was the following:

does there exist a simple and general principle that, on the basis of classical physics, explains in a convincing way, how a deterministic, classical, dynamical system can give rise to non Kolmogorovian statistics in the same way in which general relativity explains, with the presence of masses, the emergency of not Euclidean geometries?

While the mathematical development and the physical applications of quantum probability, from the early 1970's to nowadays, has been impetuous, the search of this new, intuitive idea was slow and laborious and was concluded only in 1993 with the intuition that adaptive dynamical systems can give rise to non kolmogoroviane statistics.

This intuition was metaphorically expressed by the the chameleon effect. This can be expressed by the statement: the dynamics of a system depends on the observable one measures.

In order to understand the implications of this simple and natural physical principle it is necessary a further mathematical development.

More precisely an extension of von Neumann theory of the quantum measurement process in which two ingredients, important for the present argument, were absent:

- locality

- causality

Moreover von Neumann's theory only regarded the quantum systems, while for the goals of quantum probability it was necessary a unified formulation, equally applicable to classical and quantum systems.

Such a generalization was formulated for the first time in the paper [Ac93]. This theory is moreover exposed in Chapter IX.7 of the book [Ac97] (Appendix (i), "Una teoria realistica della misura"). The theorem exposed in section (4.2) of the present paper and the experiment, which is nothing but the computer simulation of this theorem, is only an example of this more general theory. 


\subsection{The chameleon effect: adaptive systems}

A first synthesis of this line of thought was realized in the book "Urns and chameleons" (cf. [Ac97]) where the chameleon metaphor in the title illustrates the prototype of adaptive system.

This book was written in a popularization style with the exception of a technical appendix explaining the mathematical mechanism through which the "chameleon effect" can give rise to non Kolmogorovian statistics.

But the solution of a theoretical problem is less convincing than a concrete example and many people, commenting the book, asked the following question:

your argument demonstrates that, in principle, it is possible to reproduce the EPR correlations using the chameleon effect. But can you pass from principle to realty? Can you reproduce effectively these correlations using this effect?

The affirmative answer to these questions was given in the 2001 simultaneously with a paper and an experiment. The paper [AcImRe01] contains the proof of a theorem, on which the experiment is based (for commodity of the reader the precise statement of the theorem and its proof has been reproduced in section (4.2).

Simultaneously with the publication of the Preprint of this paper, a program that allowed to reproduce the EPR correlations with local and independent choices of three personal computers (classical apparata for excellence) was made available on the web page of the Volterra Center.

This theorem and this experiment provide the conclusive confirmation of the thesis of the quantum probability, advocated in the book [Ac97]: there exists no incompatibility between quantum theory, realism and relativity theory.

\subsection{Appendix: The EPR-chameleon experiment}

This section describes the classical, adaptive, deterministic, reversible, dynamical system, constructed in [AcImRe01] which reproduces EPR correlations in full respect of the locality condition.

Notice that the assumptions of the theorem contain no artificial post-selection procedures.

The experiment confirms that this dynamical system can effectively to be simulated with local and independent operations.

This new class of dynamical systems is very interesting and has potential applications to physics, biology, medicine and economy.

A different class of examples was later built in the paper [KhrSmoTru05].

Definition 2 Given:

- a measurable space (state space)

$$
\left(\Omega_{1} \times \Omega_{2}, \mathcal{F}_{1} \times \mathcal{F}_{2}\right)
$$

- for every $a, b \in[0,2 \pi)$ two functions (observable) with values \pm 1

$$
\begin{aligned}
& S_{a}^{(1)}: \omega_{1} \in \Omega_{1} \rightarrow S_{a}^{(1)}\left(\omega_{1}\right) \in\{+1,-1\} \\
& S_{b}^{(2)}: \omega_{2} \in \Omega_{2} \rightarrow S_{b}^{(2)}\left(\omega_{2}\right) \in\{+1,-1\}
\end{aligned}
$$

- for every $a, b \in[0,2 \pi)$ two invertible functions (local dynamics)

$$
T_{1, a}: \Omega_{1} \rightarrow \Omega_{1} \quad ; \quad T_{2, b}: \Omega_{2} \rightarrow \Omega_{2}
$$

The (local, deterministic, reversible, classical, adaptive) dynamical system

$$
\left\{\left(\Omega_{1} \times \Omega_{2}, \mathcal{F}_{1} \times \mathcal{F}_{2}, P\right), T:=T_{1, a} \otimes T_{2, b}\right\}
$$

is said to realize the EPR correlations if

$$
\left\langle S_{a}^{(1)} S_{b}^{(2)}\right\rangle:=\iint_{\Omega_{1} \times \Omega_{2}} S_{a}^{(1)}\left(\omega_{1}\right) S_{b}^{(2)}\left(\omega_{2}\right) d P \circ T^{-1}\left(\omega_{1}, \omega_{2}\right)=-\cos (b-a)
$$

The dynamical (classic, deterministic, local) system that we will consider is composed from two spacially separated sub-systems $\left(1, M_{1}\right)$ and $\left(2, M_{2}\right)$, interpreted as follows:

-1 and 2 are particles

- $M_{1}$ and $M_{2}$ they are measurement apparata

- the state space of $\left(1, M_{1}\right)$ is $[0,2 \pi) \times \mathbb{R}$ and also the state space of $\left(2, M_{2}\right)$ is $[0,2 \pi) \times \mathbb{R}$

- therefore the state space of the entire system $\left(1, M_{1}, 2, M_{2}\right)$ is $[0,2 \pi)^{2} \times \mathbb{R}^{2}$

The (local, adaptive) dynamics $T_{1, a}, T_{2, b}$ where 1,2 are indices for particles and $a, b$ indices for the apparata, is defined by the following conditions:

$$
T_{1, a}, T_{2, b}[0,2 \pi) \times \mathbb{R} \rightarrow[0,2 \pi) \times \mathbb{R}
$$




$$
\begin{gathered}
T_{1, a}\left(\sigma_{1}, \lambda_{1}\right):=\left(s_{1, a}\left(\sigma_{1}, \lambda_{1}\right), m_{1, a}\left(\sigma_{1}, \lambda_{1}\right)\right) \quad ; \quad T_{2, b}\left(\sigma_{2}, \lambda_{2}\right):=\left(s_{2, b}\left(\sigma_{2}, \lambda_{2}\right), m_{2, b}\left(\sigma_{2}, \lambda_{2}\right)\right) \\
s_{1 a}\left(\sigma_{1}, \lambda_{1}\right):=\sigma_{1}, \quad ; \quad m_{1, a}\left(\sigma_{1}, \lambda_{1}\right):=\lambda_{1} \frac{1}{T_{1, a}^{\prime}\left(\sigma_{1}\right)} \\
s_{2, b}\left(\sigma_{2}, \lambda_{2}\right):=\sigma_{2} \quad ; \quad m_{2, b}\left(\sigma_{2}, \lambda_{2}\right):=\lambda_{2} \frac{1}{T_{2, b}^{\prime}\left(\sigma_{2}\right)}
\end{gathered}
$$

and the functions

$$
T_{1, a}^{\prime}, T_{2, b}^{\prime} \quad[0,2 \pi] \rightarrow[0,2 \pi]
$$

are defined by

$$
T_{1, a}^{\prime}\left(\sigma_{1}\right)=\frac{\sqrt{2 \pi}}{4}\left|\cos \left(\sigma_{1}-a\right)\right| \quad, \quad T_{2, b}^{\prime}\left(\sigma_{2}\right)=\sqrt{2 \pi} \quad, \sigma_{1}, \sigma_{1} \in[0,2 \pi]
$$

In other words the adaptive dynamics (8) are given by:

$$
T_{1, a}\left(\sigma_{1}, \lambda_{1}\right):=\left(\sigma_{1}, \frac{\lambda_{1} 4}{\sqrt{2 \pi}}\left|\cos \left(\sigma_{1}-a\right)\right|\right) \quad ; \quad T_{2, b}\left(\sigma_{2}, \lambda_{2}\right):=\left(\sigma_{2}, \frac{\lambda_{2}}{\sqrt{2 \pi}}\right)
$$

where $\sigma_{1}, \sigma_{1} \in[0,2 \pi) ; \lambda_{1}, \lambda_{2} \in \mathbb{R}$.

For $\sigma_{1}, \sigma_{2} \in[0,2 \pi), \lambda_{1}, \lambda_{2} \in \mathbb{R}$ and in the notations (9), (10), we define the local measures

$$
\begin{gathered}
p_{S}\left(d \sigma_{1}, d \sigma_{2}\right)=\frac{1}{2 \pi} \delta\left(\sigma_{1}-\sigma_{2}\right) d \sigma_{1} d \sigma_{2} \\
p_{1, a}\left(\sigma_{1}, d \lambda_{1}\right)=\delta\left(m_{1, a}\left(\sigma_{1}, \lambda_{1}\right)-m_{a}\right) d \lambda_{1} \\
p_{2, b}\left(\sigma_{2}, d \lambda_{2}\right)=\delta\left(m_{2, b}\left(\sigma_{2}, \lambda_{2}\right)-m_{b}\right) d \lambda_{2}
\end{gathered}
$$

where $m_{a}, m_{b}$ they are arbitrary real numbers.

Remark (1) $p_{S}\left(d \sigma_{1}, d \sigma_{2}\right)$ is the preparation of the composite system $(1,2)$ at the source and, for the principle of causality, it cannot depend on the preparation of the apparatus.

In fact at time $t=0$ the particles cannot know which will be the preparation of the apparatus at time $t=1$ (the first moment of interaction with it).

$p_{1, a}\left(\sigma_{1}, d \lambda_{1}\right)$ and $p_{2, b}\left(\sigma_{2}, d \lambda_{2}\right)$ are the initial preparations of the local apparata. They are preparations of "response-type" and must be interpreted in the adaptive sense, i.e.: if, at time $t=1$, the particle $x(x=1,2)$ will arrive to the apparatus $M_{x}$ in the state $\sigma_{x}$, then the local contribution of this interaction to the global dynamics is described by the factor $p_{1, x}\left(\sigma_{x}, d \lambda_{x}\right)$ (cf. [AcImRe0] where it is proved how the theory of adaptive dynamical systems naturally leads to measures of this type). Combining together the above described local measures, we define the measure (initial distribution) on $[0,2 \pi)^{2} \times \mathbb{R}^{2}$ :

$$
p_{S}\left(\sigma_{1}, \sigma_{2}\right) p_{1, a}\left(\sigma_{1}, \lambda_{1}\right) p_{2, b}\left(\sigma_{2}, \lambda_{2}\right) d \sigma_{1} \sigma_{2} d \lambda_{1} d \lambda_{2}
$$

Finally, for $a, b$ as above, we define the \pm 1 valued functions (observable)

$$
S_{a}^{(1)}, S_{b}^{(2)}[0,2 \pi] \times \mathbb{R} \rightarrow\{ \pm 1\}
$$

by means of

$$
S_{a}^{(1)}(\sigma, \mu)=S_{a}^{(1)}(\sigma)=\operatorname{sgn}(\cos (\sigma-a)) \quad, \quad S_{b}^{(2)}=-S_{b}^{(1)} \quad ; \quad \sigma \in[0,2 \pi), \mu \in \mathbb{R}
$$

Theorem 2 In the above notations, the measure (13) is a probability measure on $[0,2 \pi)^{2} \times \mathbb{R}^{2}$. Moreover

$$
\begin{gathered}
\int S_{a}^{(1)}\left(s_{1, a}\left(\sigma_{1}, \lambda_{1}\right), m_{1, a}\left(\sigma_{1}, \lambda_{1}\right)\right) S_{b}^{(2)}\left(s_{2, b}\left(\sigma_{2}, \lambda_{2}\right), m_{2, b}\left(\sigma_{2}, \lambda_{2}\right)\right) \cdot p_{S}\left(\sigma_{1}, \sigma_{2}\right) p_{1, a}\left(\sigma_{1}, \lambda_{1}\right) p_{2, b}\left(\sigma_{2}, \lambda_{2}\right) d \sigma_{1} d \sigma_{2} d \lambda_{1} d \lambda_{2}= \\
=-\cos (a-b)
\end{gathered}
$$

In other words the dynamical system

$$
\left\{[0,2 \pi)^{2} \times \mathbb{R}^{2}, \text { Borel }, p_{S}\left(\sigma_{1}, \sigma_{2}\right) p_{1, a}\left(\sigma_{1}, \lambda_{1}\right) p_{2, b}\left(\sigma_{2}, \lambda_{2}\right) d \sigma_{1} \sigma_{2} d \lambda_{1} d \lambda_{2}, T_{1, a} \otimes T_{2, b}\right\}
$$

with $T_{1, a}$ and $T_{2, b}$ given by (12) realizes the EPR correlations. 
Proof. The positivity is obvious.

In order to verify (16) notice that, with the choices (15), (13), the correlations (16) become

$$
-\int_{0}^{2 \pi} \int_{0}^{2 \pi} \int_{\mathbb{R}} \int_{\mathbb{R}} \operatorname{sgn}\left(\cos \left(\sigma_{1}-a\right)\right) \operatorname{sgn}\left(\cos \left(\sigma_{1}-b\right)\right) \frac{1}{2 \pi} \delta\left(\sigma_{1}-\sigma_{2}\right) d \sigma_{1} d \sigma_{2} \delta\left(\frac{\lambda_{1}}{T_{1, a}^{\prime}\left(\sigma_{1}\right)}-m_{a}\right) \delta\left(\frac{\lambda_{2}}{T_{1, b}^{\prime}\left(\sigma_{2}\right)}-m_{b}\right) d \lambda_{1} d \lambda_{2}
$$

Using the identity

$$
\int_{\mathbb{R}} \delta(a \lambda-m) d \lambda=\frac{1}{a} \int_{\mathbb{R}} \delta\left(\lambda-\frac{m}{a}\right) d \lambda
$$

and denoting

$$
m_{a}^{\prime}:=T_{1, a}^{\prime}\left(\sigma_{1}\right) m_{a} \quad ; \quad m_{b}^{\prime}:=T_{2, b}^{\prime}\left(\sigma_{2}\right) m_{b}
$$

After this change of variable (17) becomes

$$
\begin{gathered}
-\int_{0}^{2 \pi} \int_{0}^{2 \pi} \int_{\mathbb{R}} \int_{\mathbb{R}} \operatorname{sgn}\left(\cos \left(\sigma_{1}-a\right)\right) \operatorname{sgn}\left(\cos \left(\sigma_{1}-b\right)\right) \frac{1}{2 \pi} \delta\left(\sigma_{1}-\sigma_{2}\right) d \sigma_{1} d \sigma_{2} \delta\left(\mu_{1}-m_{a}^{\prime}\right) \delta\left(\mu_{1}-m_{b}^{\prime}\right) T_{1, a}^{\prime}\left(\sigma_{1}, \mu_{1}\right) d \mu_{1} T_{2, b}^{\prime}\left(\sigma_{2}, \mu_{2}\right) d \mu_{2} \\
=-\frac{1}{2 \pi} \int_{0}^{2 \pi} \int_{\mathbb{R}} \int_{\mathbb{R}} \operatorname{sgn}(\cos (\sigma-a)) \operatorname{sgn}(\cos (\sigma-b)) d \sigma \delta\left(\mu_{1}-m_{a}\right) \delta\left(\mu_{1}-m_{b}\right) T_{1, a}^{\prime}\left(\sigma, \mu_{1}\right) d \mu_{1} T_{2, b}^{\prime}\left(\sigma, \mu_{2}\right) d \mu_{2} \\
=-\frac{1}{2 \pi} \int_{0}^{2 \pi} \int_{\mathbb{R}} \int_{\mathbb{R}} \operatorname{sgn}(\cos (\sigma-a)) \operatorname{sgn}(\cos (\sigma-b)) d \sigma \delta\left(\mu_{1}-m_{a}^{\prime}\right) \delta\left(\mu_{1}-m_{b}^{\prime}\right) \frac{\sqrt{2 \pi}}{4}|\cos (\sigma-a)| d \mu_{1} \sqrt{2 \pi} d \mu_{2} \\
=-\frac{1}{4} \int_{0}^{2 \pi} \operatorname{sgn}(\cos (\sigma-a)) \operatorname{sgn}(\cos (\sigma-b)) d \sigma|\cos (\sigma-a)|=-\frac{1}{4} \int \cos (\sigma-a) \operatorname{sgn}(\cos (\sigma-b)) d \sigma=-\cos (b-a)
\end{gathered}
$$

which is (16). Finally the normalization condition

$$
\int p_{S}\left(\sigma_{1}, \sigma_{2}\right) p_{1, a}\left(\sigma_{1}, \lambda_{1}\right) p_{2, b}\left(\sigma_{2}, \lambda_{2}\right) d \sigma_{1} \sigma_{2} d \lambda_{1} d \lambda_{2}=1
$$

leads, with a calculation similar to the above one, to the identity

$$
\frac{1}{4} \int_{0}^{2 \pi}|\cos (\sigma-a)| d \sigma=1
$$

which is easily verified.

\section{Conclusions}

Let us now come back to the question posed in the title of the present paper:

would Einstein be convinced that the theorems and the experiments realized in the past 30 years by quantum probability solve the interpretative paradoxes of quantum theory and reconcile the points of view of Einstein with those of Bohr and Heisenberg?

Einstein's main insistence was on the notion of element of reality and of pre-determination. On the other hand Bohr's main insistence was on the active role of measurement which, in some sense, determines the reality and Heisenberg emphasized the role of observable data.

The chameleon metaphor combines in a satisfactory way these two points of view: the color of the chameleon is pre-determined (green on the leaf, brown on the wood) and at the same time it is also created by the measurement, i.e. by the choice of the leaf or of the wood.

Thus the compatibility of the classical and the quantum world does not come out from artificial, purely formal, constructions but from a simple although non trivial physical intuition.

Realism is saved, but the passive realism of the ballot box must be ampliated to the adaptive realism of the chameleon.

The mathematical translation of this metaphor, realized in the construction of Theorem (2) in section (4.2) shows that the theory of adaptive systems is rich enough to reproduce, in full agreement with the principles of causality and locality, the notorious EPR correlations.

More generally we can say that: adaptive systems can produce non-Kolmogorovian statistics, i.e. statistical data which cannot be described within a single classical probabilistic model.

Following Heisenberg's prescription the discrimination between the two probabilistic models is entirely based on observable data through the statistical invariants.

A posteriori the incompatibility of the classical probabilistic models, corresponding to different contexts, may be hardly surprising. But what is surely surprising is that the more powerful quantum probabilistic model can provide a unified mathematical framework for all these contexts. 
Finally the most distinctive among the physical principles of quantum theory, Heisenberg indeterminacy principle, is proved (see [Ac95a]) to imply all those features of the quantum model which seemed particularly far from the intuition: the Hilbert space, the superposition principle, the complex numbers, amplitudes instead of probabilities, etc. ...

Thus now we can claim that the mathematical structure of QM is not a kind of black box that we use because, for mysterious reasons, it works, but a conceptual necessity which follows from the physical requirements of the theory.

This intrinsic harmony between the physical requirements and the corresponding mathematical structures, this replacement of magical collapses and associated spooky action at distance by the physically plausible distinction between response statistics and passive statistics, would have surely appealed the esthetic feeling of Einstein who always maintained that a good theory is much more than a set of empirical working rules.

\section{Bibliography}

[Ac81a] Accardi L.: Topics in quantum probability, Phys. Rep. 77 (1981) 169-192

[Ac83a] Accardi L.: The probabilistic roots of the quantum mechanical paradoxes, In: The wave-particle dualism, S. Diner, G. Lochak, and F. Selleri (eds.) Reidel, Dordrecht (1984) 297-330

[Ac81b] Accardi L.: Probabilità e teoria quantistica. Physis 23 (1981) 485-524

[AcFe81] Accardi L., Fedullo A.: On the statistical meaning of complex numbers in quantum theory, Lettere al Nuovo Cimento 34 (1982) 161-172 University of Salerno preprint May (1981)

[Ac81d] Accardi L.: Quantum theory and non kolmogorovian probability, In: Stochastic processes in quantum theory and statistical physics, ed. S. Albeverio et al., Springer LNP n.173 (1982) 1-18

[Ac82a] Accardi L.: Foundations of quantum probability. Rendiconti del Seminario Matematico dell'Università e del Politecnico, Torino (1982) 249-273

[Ac82c] Accardi L.: Some trends and problems in quantum probability, In: Quantum probability and applications to the quantum theory of irreversible processes, L. Accardi, A. Frigerio and V. Gorini (eds.) Springer LNM n. 1055 (1984) 1-19

[Ac83a] Accardi L.: The probabilistic roots of the quantum mechanical paradoxes. In : The wave-particle dualism, S. Diner, G. Lochak, and F. Selleri (eds.) Reidel, Dordrecht (1984) 297-330

[Ac85] L. Accardi:

Non kolmogorovian probabilistic models and quantum theory. invited talk given by L. Accardi the 45-th ISI Conference, Discussant J.S. Watson, Amsterdam, August (1985), ISI BulletiN. rielaborated by: [AcRe94] L. Accardi and E. Reviglio under the title: Quantum Probability and the Interpretation of Quantum Theory. Volterra Preprint N. 166 (1994)

[Ac86] Accardi L.: Foundations of Quantum Mechanics : a quantum probabilistic approach. in The Nature of Quantum Paradoxes; eds. G.Tarozzi, A. van der Merwe Reidel (1988) 257-323 Preprint Dipartimento Di Matematica, Università di Roma Torvergata (1986)

[Ac93] L. Accardi: Einstein-Bohr: one all, in: The interpretation of quantum theory: where do we stand? Acta Enciclopedica, Istituto dell'Enciclopedia Italiana (1994) 95-115 Volterra preprint N. 174 (1993)

[Ac93] L. Accardi: On the axioms of probability, in: The Foundations of Quantum Mechanics, Proceedings of the Lecce Conference (1993) C. Garola, A. Rossi (eds.) Kluwer (1995) 1-18 Volterra Preprint N. 194 November 1994

[Ac95a] Accardi L.: Can mathematics help solving the interpretational problems of quantum theory? Il Nuovo Cimento 110 B (1995) 685-721 Volterra Preprint N. 203 (1995)

[Ac97] Luigi Accardi: Urne e camaleonti. Dialogo sulla realtà, le leggi del caso e la teoria quantistica. Il Saggiatore (1997); russian edition, Regular and Chaotic dynamics (2003); japanese edition, ed. Makino (2003); English edition (traduzione di Daniele Tartaglia), World Scientific (2003)

[AcLuVo02] Accardi L., Y.G. Lu, I. Volovich: Quantum Theory and its Stochastic Limit. Springer Verlag (2002) Japanese translation, Tokyo-Springer (2002)

[AcKo00b] L. Accardi, S.V. Kozyrev: Quantum interacting particle systems, Lectures given at the: VolterraCIRM International School: Quantum interacting particle systems , Levico Terme, 23-29 September 2000, in: Accardi L., F. Fagnola (eds.) "Quantum interacting particle systems", World Scientific (2002) Preprint Volterra, N. 431 September (2000)

[QP - PQ] Series: Quantum Probability and Related Topics: [QP-0] (1978), [QP-I, II, III] Springer LNM N.1055 (1984), N. 1136 (1986), N. 1033 (1987), N. 1396 (1989), N. 1442 (1990); [QP-VI, .., XVII] World Scientific $(1991, \ldots, 2003)$

[AcImRe01] L.Accardi, K.Imafuku, M.Regoli: On the physical meaning of the EPR-chameleon experiment, Infinite dimensional analysis, quantum probability and related topics, 5 N. 1 (2002) 1-20 quant-ph/0112067 
Volterra Preprint N. 494 December (2001) N. 494

[AcImRe03a] Luigi Accardi, , Kentaro Imafuku, Massimo Regoli: Adaptive dynamical systems and the EPRchameleon experiment, in: Proceedings of the Conference: Foundation of Probability and Physics-2, Vaxjo, June 2-7 (2002), to appear (2003)

[AcFi03] L. Accardi, F. Fidaleo: Entangled Markov chains. Annali di Matematica Pura e Applicata (2004) Preprint Volterra N.556 (2003) http://www.springerlink.com/index/10.1007/s10231-004-0118-4

[Acc04] L. Accardi: Some loopholes to save quantum nonlocality, in: Proceedings of the conference: Foundations of Probability and Physics-3, Vaxjo, Sweden 7-12 june (2004), Andrei Khrennikov (ed.), American Institute of Physics, AIP Conference Proceedings 750 (2004) 1-20

[Belt91] Beltrametti E.: On the problem of classical and non-classical probabilities. J. Math. Phys. 32 (5) (1991) 1280-1286

[Belt92] Beltrametti E.: Recent facts in quantum logic surroundings. Proceedings Symposium on the Foundations of quantum mechanics, Colonia

[BeDelNMa93] Beltrametti E., C. Del Noce, M.J. Maczynsky, Characterization and deduction of Bell-type inequalities. in: The foundations of quantum mechanics - Historical analysis and open questions, Lecce 1993, ed. C.Garola, A. Rossi, Kluwer (1995)

[BeCa94] Beltrametti E.G., Cassinelli G.: Quantum Measurement and Probability, Ed. L. Accardi, Istituto della Enciclopedia Italiana, novembre (1994)

[Bengt03] Ingemar Bengtsson: The importance of being unistochastic, in: Proceedings of the conference: Quantum theory: Reconsideration of the Foundations-2, Vaxjo, Sweden 1-7 june (2003), Andrei Khrennikov (ed.), Vaxjo University Press (2004) 93-100

[Bengt04] Ingemar Bengtsson: MUB's, polytopes and finite geometries, in: Proceedings of the conference: Foundations of Probability and Physics-3, Vaxjo, Sweden 7-12 june (2004), Andrei Khrennikov (ed.), American Institute of Physics, AIP Conference Proceedings 750 (2004) 63-69

[CasVar02] Cassinelli G., Varadarajan V.S.: On Accardi's notion of complementary observables, Infinite Dimensional Analysis, Quantum Probability and Related Topics 5 (2) (2002) 135-144

[DBStr01] De Baere W., Struyve W.: Subquantum nonreproducibility and the complete local description of physical reality Preprint (2001)

[DMDBMa01] de Muynck W.M., De Baere W., Martens H.: Interpretations of quantum mechanics, joint measurement of incompatible observables, and counterfactual definiteness Foundations of Physics, 24, n. 12 (1994) 1589-1663

[Feyn51] Feynmann R.P. The concept of probability in quantum mechanics. in :Proc. II-d Berkeley Sym on Math. Stat. and Prob. ; Univ. of California Press , Berkeley, Los Angeles (1951) 533-541

[FeLeSa66] Feynmann R.P., Leighton R.P., Sands M.: Lectures on Physics, III, Addison-Wesley (1966)

[FilSvoz04] S. Filipp, K. Svozil: Tracing the bounds on Bell-type Inequalities, in: Proceedings of the conference: Foundations of Probability and Physics-3, Vaxjo, Sweden 7-12 june (2004), Andrei Khrennikov (ed.), American Institute of Physics, AIP Conference Proceedings 750 (2004) 1-20

[Fine82a] Fine A.: Hidden Variables, Joint Probability, and the Bell Inequalities Physical Review Letters, 48 no. $5(1982)$

[GutkVaFr84] Gutkowski D., Valdes Franco M.V.: Kolmogorovian statistical invariants for the Aspect Rapisarda experiment, SLNM 1055 [QP-V] (ed) Accardi L., Frigerio A., Gorini V. (1984) 146-152

[Gust00] Karl Gustafson: Quantum Trigonometry, IDA-QP 3 (2000) 33-52

[Gust02] Karl Gustafson: Bell's inequality and the Accardi-Gustafson inequality, in: Proceedings of the Conference: Foundation of Probability and Physics-2, Vaxjo, June 2-7 (2002) 202-223

[HePhi01a] Hess K., Philipp W.: A possible loophole in the Bell's theorem and the problem of decidability between the views of Einstein and Bohr, PNAS 9825 (2001) 14228-14233

[HePhi01b] Hess K., Philipp W.: A possible loophole in the theorem of Bell, PNAS 9825 (2001) 14224-14227

[Jamm74] Jammer M. The philosophy of quantum mechanics. Wiley (1974)

[KhrSmoTru05] Khrennikov A., O.G. Smolyanov, A. Truman: Kolmogorov probability spaces describing Accardi models of quantum correlations, Open Systems and Information Dynamics (2005)

[Khre02] Khrennikov A.: Vaxjo interpretation of quantum mechanics, in: Proceedings of the Conference: Quantum theory: Reconsideration of the Foundations-2, Vaxjo, June 2-7 (2002), Reports from MSI n. 20018 (2002)

[Kolmo33] Kolmogorov A.N.: Grundbegriffe der Wahrscheinlichkeitsrechnung. Springer Verlag, Berlin (1933); reprinted: Foundations of the Probability Theory, Chelsea Publ. Comp., New York (1950), (1956)

[LahtYli87] Lahti P.J., Ylinen K.: On total noncommutativity in quantum mechanics, J. Math. Phys. 28 (11) (1987) 2614-2617

[Lahti85] Lahti P.J.: Uncertainty complementarity and commutativity, Preprint 1985 
[MaasUff88] Maassen H., Uffink J.: Generalized entropic uncertainty relations, Phys. Rev. Lett. 60 (1988) 1103-1106

[Merm82a] Mermin N.D.: Joint Distributions and Local Realism in the Higher-Spin Einstein-PodolskyRosen Experiment, Reprinted from Foundations of physics 12 (1982) 101-135

[Merm82b] Mermin N.D.: Comment on "Hidden Variables, Joint Probability, and the Bell Inequalities", Physical review letters 49 (1982) 242-243

[Mey96] Meyer D. A.: From quantum cellular automata to quantum lattice gases, J. Stat. Phys. 85 (1996) $551-574$

[Miyad05] Takayuki Miyadera: Entangled Markov Chains generated by Symmetric Channels, Infinite Dimensional Analysis, Quantum Probability and Related Topics 8, No. 3 (2005)

[Pito94] Pitowsky I.: George Boolès 'conditions of possible experiencè and the quantum puzzle, Brit. J. Phil. Sci. 45 (1994), 95-125

[Pito89] Pitowsky I.: Quantum probability and quantum logic, Springer LNP N. 321 (1989)

[Volo00b] Volovich I.V.: Quantum cryptography in space and Bell's theorem, in: Proceedings of the conference: Foundations of Probability and Physics-1, Vaxjo, Sweden 25 November - 1 december (200), Andrei Khrennikov (ed.), A. Khrennikov (ed.), World Scientific (2001) 364-371

[vNeum32] J. von Neumann: Mathematical foundations of Quantum Mechanics, Princeton University Press (1955) also: Mathematische Grundlagen der Quantenmechanik, Berlin, Springer (1932), Dover-Usa (1943) 